\title{
Closing the gap between practice and science in school- and community-based participatory physical literacy promotion: study protocol of the StuPs project
}

Stefanie Wessely ${ }^{1 *}$ D, Dagmar Starke ${ }^{2}$, Simone Weyers ${ }^{3}$ and Christine Joisten ${ }^{1}$

\begin{abstract}
Background: The role of physical activity in the promotion of children's well-being and health is widely known. However, research indicates that the time spent physically exercising and participating in organized sport activities is decreasing among children. Although there is currently no gold standard for promoting sustainable physical activity in children, community-based approaches, particularly those that are multicomponent, appear to be the most successful. The project StuPs: a school- and community-based participatory approach for promoting physical activity in children and their families aims to develop a community-based approach to promoting physical activity by increasing physical literacy among elementary school children and their household members.
\end{abstract}

Methods: The project is built upon the intervention mapping approach and consists of two periods with an overall duration of 3 years. Period I will last 9 months and include an assessment of needs, wants, strengths, and weaknesses regarding physical activity and health promotion at the community- and school-based level according to the keywords "capacity building" and "physical literacy." Based on the knowledge gained in this stage, measures for capacity building to promote healthy lifestyles and physical literacy in children will be developed using the community-based participatory research and capacity building approach. In Period II, the measures will be applicated, implemented and evaluated using a pre-/post-design to assess efficacy.

Discussion: Although the efficacy of using community-based and capacity building approaches to reach children is promising, there remains a gap regarding best practices for changing existing structures and habits over the long term and in the sense of promoting physical literacy.

Keywords: Physical activity promotion, Physical literacy, Socially deprived urban districts, Community-based approach, Community-based participatory research, Capacity building

\section{Background}

Increasingly sedentary behavior in children constitutes a growing global challenge, although awareness of the role of physical activity in optimizing children's health is

\footnotetext{
* Correspondence: s.wessely@dshs-koeln.de

1 Department for Physical Activity in Public Health, Institute of Movement and Neurosciences, German Sport University Cologne, Am Sportpark Müngersdorf 6, 50933 Cologne, Germany

Full list of author information is available at the end of the article
}

ubiquitous [1, 2]. Despite the various health benefits of physical activity on physical, psychosocial, cognitive, motor skill, and language development [3], only $20 \%$ of children and adolescents worldwide meet the World Health Organization (WHO)'s physical activity recommendations of $60 \mathrm{~min}$ per day [2]. Similar data resulted from a German health survey from 2014 to 2017 conducted by the Robert Koch Institute: only $22.4 \%$ of girls

(c) The Author(s). 2021 Open Access This article is licensed under a Creative Commons Attribution 4.0 International License, which permits use, sharing, adaptation, distribution and reproduction in any medium or format, as long as you give appropriate credit to the original author(s) and the source, provide a link to the Creative Commons licence, and indicate if changes were made. The images or other third party material in this article are included in the article's Creative Commons licence, unless indicated otherwise in a credit line to the material. If material is not included in the article's Creative Commons licence and your intended use is not permitted by statutory regulation or exceeds the permitted use, you will need to obtain permission directly from the copyright holder. To view a copy of this licence, visit http://creativecommons.org/licenses/by/4.0/. The Creative Commons Public Domain Dedication waiver (http://creativecommons.org/publicdomain/zero/1.0/) applies to the data made available in this article, unless otherwise stated in a credit line to the data. 
and $29.4 \%$ of boys met the WHO's recommendations [4] - a far cry from the German physical activity recommendations of $90 \mathrm{~min}$ or more per day among elementary school children [5]. These data have also shown that time spent physically exercising correlates positively with socioeconomic status and negatively with age. Therefore, the challenge remains to develop efficient and sustainable strategies for promoting physical activity among children. The StuPs project: a school- and communitybased participatory approach for promoting physical activity in children and their families, was developed for this purpose, particularly in response to "Bewegung und Bewegungsförderung" ("exercise and physical activity promotion"), the German Federal Ministry of Health's call for funding [6]. The aim of this call was to spread the knowledge of the German recommendations for physical activity in the sense of health in all policies. Currently, there is no gold standard for promoting physical activity in young children, especially among vulnerable groups; despite this, school-based and multicomponent approaches are suspected to be most efficient. Because schools are the optimal environment for reaching a great number of youth demographics, school-based approaches seem to have high efficacy [5]. However, physical activity promotion in schools seems to have a limited impact on children's movement behavior during their leisure time [7]. Current evidence assumes greater success in preventing childhood obesity if entire communities are involved in creating and promoting healthy environments [8]. The inclusion of additional out-ofschool aspects within the scope of multicomponent approaches and the inclusion of families, communities, policies, and stakeholders in a participatory manner is recommended [5, 7]. Participatory approaches, such as community-based participatory research (CBPR) [9], have the potential to generate synergy, reach greater acceptance within the target group, and engender increased engagement $[8,10]$, especially in combination with capacity building $[8,11]$. On a behavioral level, the available evidence supports an approach that promotes physical literacy, a construct that indicates the basis for an active lifestyle and serves as "a primary determinant of health and disease" [12]. Physical literacy thus focuses on skill acquisition. In this way, it is similar to the construct of health literacy, which inculcates the ability to find, understand, evaluate, and apply health information and therefore combines knowledge with motivation and competence $[13,14]$. To date, no intervention combining physical literacy with community-based strategies has been conducted. Therefore, the StuPs project aims to close this gap between science and practice by implementing a participatory and interdisciplinary multicomponent approach targeting both schools and communities in two socially deprived areas in Cologne
(North Rhine-Westphalia, Germany) within the scope of capacity building for physical literacy promotion.

\section{Methods \\ Study design}

The StuPs design (Fig. 1) is intended to function as a temporary supporter of the selected districts over the project duration of three years, divided into two periods. The first period (Period I) will be conducted over the course of 9 months. Using a multicomponent, mixedmethods approach, it will identify structures like geographic information system data (GIS), existing capacities, collaborations, and networks to detect needs and wants regarding physical activity promotion in children. At the end of this period, required actions will be deduced. With the knowledge of these required actions, measures to create healthy environments in the sense of capacity building and physical literacy promotion in children will be developed. This procedure will be conducted with the participation of key stakeholders in the two Cologne districts. The intention is to create measures integrable into the routines of the stakeholders, schools, and target group and address individuals as well as their environments. Therefore, these measures will on the one hand address the behaviors of children and their families as well as their districts' capacities to create healthy environments. On the other hand, they will enable local stakeholders within the topic of health and physical literacy promotion by qualification trainings.

The intention of the second period (Period II) is to implement the developed measures over a 27-month period. Measures will be combined with existing healthrelated structures in terms of health in all policies. To evaluate efficacy, the status quo will be analyzed in a manner equal to that in Period I to capture changes on structural levels. To investigate behavioral changes, preand post-tests of the children's physical literacy levels will be conducted. Additionally, the German Federal Ministry of Health has employed an external evaluation group [6], to investigate promoters and inhibitors during the StuPs project in the context of the call for funding "Bewegung und Bewegungsförderung". The aim of the external evaluation is to detect factors of success within community-based approaches. At the end of Period II, a transfer guideline will be developed through an iterative and participatory process to be provided to various districts and communities; this transfer guideline will contain step-by-step instructions, serving as a best practice model, using as few resources as possible. Additionally, it will include information on how to i) capture needs and wants in terms of capacity building and physical literacy promotion in settings, districts, or communities; ii) apply and implement measures; ii) evaluate efficacy; and iv) document procedures to facilitate learning from each 


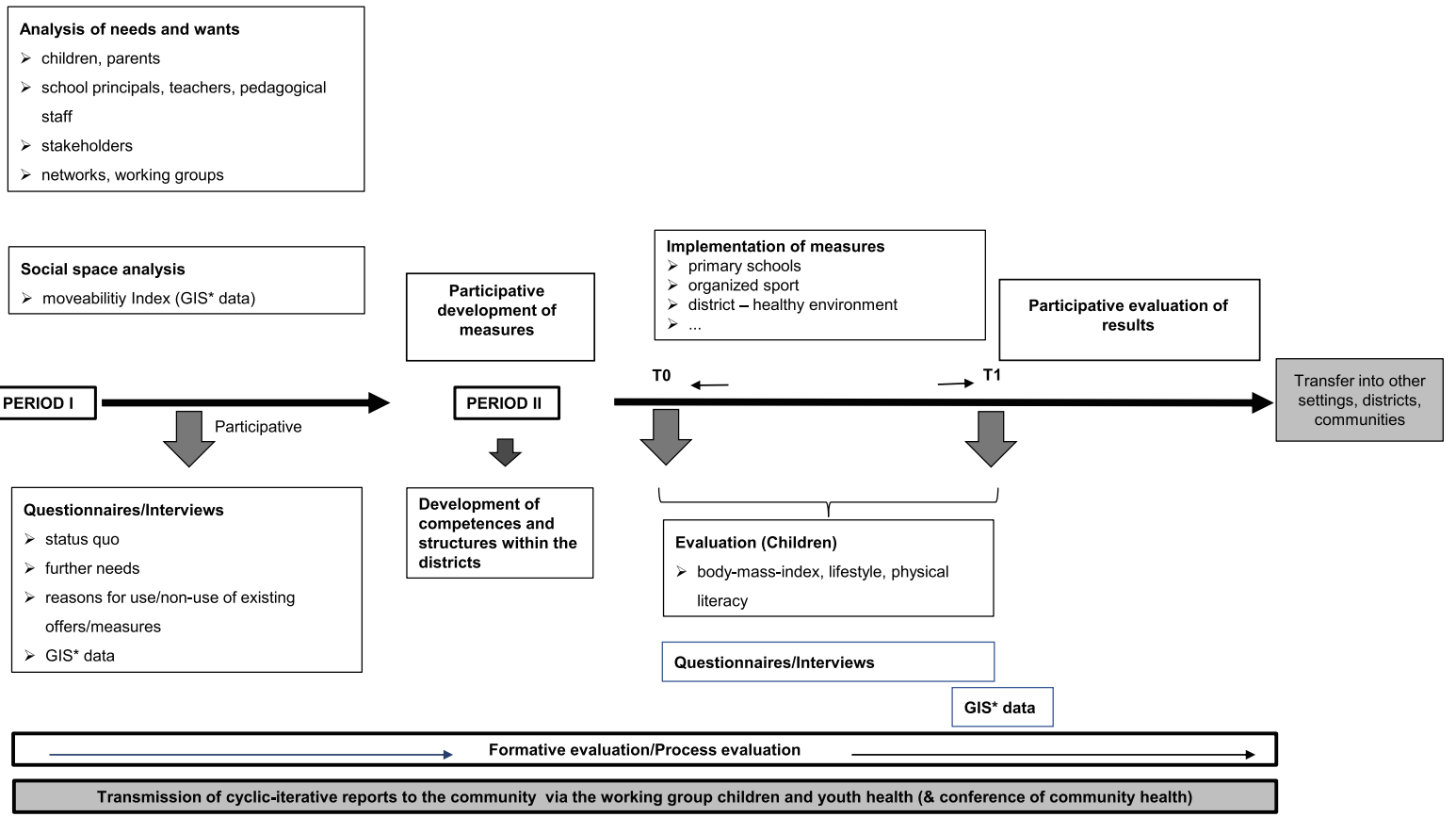

"GIS= Geographic information system

Fig. 1 Study Design of the StuPs Project

other regarding practical experiences. Throughout the entirety of the project, there will be cyclical, iterative reports and dialogues at the community level.

\section{Participants and recruitment}

The approach will be partially randomized. Participating schools from two Cologne districts (Chorweiler and Meschenich) will be recruited for this study. The two districts have been chosen because of their social circumstances, as defined by the Cologne Office for Urban Development and Statistics [15]. The index for social circumstances of urban districts is based on information related to issues such as economic deprivation, political and cultural disadvantage, and health inequality. With the support of the district coordinators responsible for social planning, two elementary schools (one in each district) will be recruited, and local networks will be identified. The elementary schools will provide access to the target group of children, parents, teachers and other pedagogical staff. Any children and parents at the chosen elementary schools will have the opportunity to participate; therefore, the target group will be composed randomly. The identified networks will be used to get in contact with local stakeholders to establish participatory collaborations.

\section{Intervention mapping}

Intervention mapping describes the combination of theory- and evidence-based approaches in a bottom-up procedure containing six steps [16]. Through these six steps, interventions can be designed that are appropriate to the target group and also consider healthy environments [17]. The StuPs project is designed to follow the six stages (Table 1). The target group will be integrated in the identification of needs, wants and development of measures. Additionally, current scientific knowledge and existing behavioral theories will be researched and considered. The six steps of the intervention mapping form the modular structure of the project and emerge in specific work packages.

Table 1 Modified Steps of Intervention Mapping within the StuPs Project [16]

Step 1: Assessment of needs, wants and inventory of health-related issues to identify current conditions in terms of capacity building and physical literacy promotion in the selected districts.

Step 2: Goal specification within the settings/networks with stakeholders in light of the knowledge generated in step 1.

Step 3: Scientific literature research and comparison with previous results of community-based approaches as well as definitions and instruments to investigate physical literacy, aiming to accompany the theoretical approach with existing knowledge.

Step 4: Derivation and development of school- and district-related measures based on the knowledge of the previous steps. In this step, the content for the measures conducted later will be created.

Step 5: Definition of further procedures and implementation of the measures created previously.

Step 6: Review, evaluation, and development of transfer indicators. 


\section{Community-based participatory research}

The CBPR approach developed by Israel et al. [9] has been decisive in designing the content of the StuPs project. CBPR aims to promote health equity, strategies development, empowerment of a target group, and capacity building within the targeted community [9]. This approach intends to combine scientific, practical, and policy stakeholders as well as different professions. Therefore, the StuPs approach is characterized by a high level of practical orientation to strengthen participatory collaborations between science, practice, and policy. To this end, the CBPR principles by Israel et al. [9] have been modified to promote individual and collective empowerment and the development of expertise and structures in terms of capacity building and physical literacy (Table 2).

\section{Data management and analysis}

Any conducted assessments will be carried out anonymously. The results of Period I will be analyzed and interpreted by conducting qualitative data analysis [18] in the MAXQDA 2020 software. The data resulting from the children and parental pre- and post-tests will be analyzed using the SPSS software version 27.0 (SPSS Inc., Chicago, IL, USA). The significance level $\alpha$ will be set at $<.05$; confidence intervals will be estimated at $95 \%$. Descriptive analysis will be conducted to identify crosssectional and long-term changes in physical literacy level, including motor skills, by generating means and variances. Additionally, correlations and influencing factors among variables will be assessed using analysis of covariance and regressions.

Table 2 Modified Principles of the Community-Based Participatory Research following Israel et al. [9]

(1) The network sees itself as a unit.

(2) The network builds on existing strengths and resources.

(3) The network works transparently and in partnership.

(4) The network promotes joint development, empowerment, and capacity building

(5) The network creates a balance between knowledge generation and interventions for the mutual benefit of all partners.

(6) The network focuses on specific needs in the context of physical activity promotion, taking into account sociodemographic and gender characteristics as well as systematic geographical data.

(7) The development of measures and implementation strategies takes place in a cyclical, iterative, and reflexive process.

(8) All results and developments will be made accessible to all partners; further dissemination and use will also take place in coordination.

(9) The aim is to create the basis for long-term cooperation and the establishment of the topics as a cross-sectional task of all partners involved (health in all policies), with the intention of sustainably promoting the physical activity (and health) and physical literacy of vulnerable groups.

\section{Discussion}

Individual habits and behaviors as well as environments (e.g., immediate surroundings, neighborhoods, schools, local policies) have a great impact on a child's health status [19]. Therefore, healthy environments need to be created, and StuPs has been developed to achieve this aim. This approach is grounded in CBPR [9] and intervention mapping [16], both of which have evidencebased efficacy e.g. in terms of anthropometric and behavioral changes as well as environmental and political outcomes $[8,16,17]$. Previous studies have reported increasing success in terms of intensity of community engagement and participation $[8,10]$. In addition, the StuPs approach has been developed following CBPR and intervention mapping to build upon existing knowledge and experiences. Although the rough procedure is comparable with other approaches, StuPs differs in its aim to improve physical literacy in children and their families. Most other community-based approaches have addressed health promotion in general or healthy weight in children specifically [8]. Currently reported physical literacy interventions have mainly been conducted in schools, especially physical education classes [20]. StuPs is unique in its aim to promote physical literacy at the community level, thereby reaching children not only where they learn but also where they live and play. This potential arises from increased scientific attention because physical literacy is assumed to be fundamental in creating physically active lifestyles [20]. According to Carney et al. [12], physical literacy is "a gateway" to lifelong physical activity and is crucial for a positive attitude toward movement. In addition to personal and individual factors such as age, gender, motor skills and psychosocial development, one's environment is assumed to impact one's degree of physical literacy [21]. The development of healthy environments - including walkable neighborhoods, opportunities to move and play, and opportunities to participate in sports and physical activity - could motivate and support children and families to develop active and therefore healthy lifestyles. For this reason, the intention of the StuPs project is to spread the knowledge of the physical literacy construct beyond schools and among community stakeholders, which, in combination with capacity building, will create healthy environments and therefore support children in developing more active lifestyles. It is expected that improved physical literacy knowledge among stakeholders will lead to increased readiness to change structures and build capacities for healthy environments for districts' children. To maintain closure of the gap between science and practice in these efforts, there will be cyclical, iterative reports at the community level within specific working groups. The members of the working groups are practical experts on different community- and district- 
related issues, and they will support the overall project duration through discussion of any barriers and obstacles that appear. The project will benefit from their expertise and experience as well as incorporate feedback and further impulses and ideas. This will be especially helpful when preparing the transfer guideline to ensure that it is as feasible as possible. With this approach, StuPs intends to contribute knowledge of supporters and barriers to promote sustainable physical activity in the scientific interest of the German Federal Ministry of Health.

\section{Abbreviations}

CBPR: Community-Based Participatory Research'; GIS: Geographic Information System; StuPs: 'Ein schul- und kommunalbasierter Ansatz zur partizipativen Bewegungsförderung von Kindern und deren Familien'; WHO: World Health Organization

\section{Acknowledgments}

Not applicable.

\section{Authors' contributions}

$\mathrm{CJ}$ is principal investigator of the StuPs project and designed the overall project in participation with SWy, DS and SW. SWy was responsible for the development of structural analysis; DS for stakeholder issues; SW drafted the manuscript together with CJ. All authors read and approved the final manuscript.

\section{Funding}

The StuPs project is funded by the German Federal Ministry of Health (BMG; grant number ZMVI1-2519FSB110, governmental funding) who had no involvement in the design of the study, or in writing of this manuscript. Open Access funding enabled and organized by Projekt DEAL.

\section{Availability of data and materials}

Data sharing is not applicable as this is a study protocol. Study materials such as the developed questionnaires or guidelines are available on reasonable request from the authors.

\section{Declarations}

\section{Ethics approval and consent to participate}

Human Ethics Research Committee ethics approval was obtained from the Ethics Commission of the German Sport University Cologne (136/2019); which examines studies considering the Declaration of Helsinki. The research will be conducted in accordance with the approved protocol. Participation requires a written declaration of informed consent; children require a written declaration by a parent or guardian. Participants will receive predefined code numbers; any personalized data will therefore be pseudo-anonymized initially, and after analysis completely anonymized. The anonymized data will remain at the study center of the German Sport University Cologne; European Data Protection Regulation of May 25th, 2018 will be complied.

\section{Consent for publication}

Not applicable.

\section{Competing interests}

The authors declare that they have no competing interests.

\section{Author details}

'Department for Physical Activity in Public Health, Institute of Movement and Neurosciences, German Sport University Cologne, Am Sportpark Müngersdorf 6, 50933 Cologne, Germany. ${ }^{2}$ Academy of Public Health Services, Kanzerlstr. 4, 40472 Düsseldorf, Germany. ${ }^{3}$ Institute of Medical Sociology, Centre for Health and Society, Medical Faculty, Heinrich-Heine-University Düsseldorf, Universitätsstraße 1, 40225 Düsseldorf, Germany.
Received: 17 March 2021 Accepted: 21 March 2021

Published online: 01 April 2021

\section{References}

1. Bremer E, Graham JD, Cairney J. Outcomes and feasibility of a 12-week physical literacy intervention for children in an afterschool program. Int J Environ Res Public Health. 2020;17(9). https://doi.org/10.3390/ijerph17093129.

2. Guthold R, Stevens GA, Riley LM, Bull FC. Global trends in insufficient physical activity among adolescents: a pooled analysis of 298 populationbased surveys with 1.6 million participants. Lancet Child Adolescent Health. 2020;4(1):23-35. https://doi.org/10.1016/S2352-4642(19)30323-2

3. Timmons BW, Leblanc AG, Carson V, Connor Gorber S, Dillman C, Janssen I, et al. Systematic review of physical activity and health in the early years (aged 0-4 years). Appl Physiol Nutr Metab. 2012;37(4):773-92. https://doi. org/10.1139/h2012-070

4. Finger JD, Varnaccia G, Borrmann A, Lange C, Mensink G. Körperliche Aktivität von Kindern und Jugendlichen in Deutschland Querschnittergebnisse aus KiGGS Welle 2 und Trends. J Health Monito. 2018;3:24-31. https://doi.org/10.17886/RKI-GBE-2018-006

5. Rütten A, Pfeifer K. Nationale Empfehlungen für Bewegung und Bewegungsförderung: Bundeszentrale für gesundheitliche Aufklärung Köln; 2017.

6. Bundesministerium für Gesundheit. https://www. bundesgesundheitsministerium.de/service/begriffe-von-a-z/b/bewegungund-bewegungsfoerderung.html. Accessed 22 Jan 2021.

7. Naylor P-J, McKay HA. Prevention in the first place: schools a setting for action on physical inactivity. Br J Sports Med. 2009;43(1):10-3. https://doi. org/10.1136/bjsm.2008.053447

8. Korn AR, Hennessy E, Tovar A, Finn C, Hammond RA, Economos CD. Engaging coalitions in community-based childhood obesity prevention interventions: a mixed methods assessment. Child Obes. 2018;14(8):537-52. https://doi.org/10.1089/chi.2018.0032

9. Israel BA, Schulz AJ, Parker EA, Becker AB. Review of community-based research: assessing partnership approaches to improve public health. Annu Rev Public Health. 1998;19(1):173-202. https://doi.org/10.1146/annurev. publhealth.19.1.173.

10. Berge JM, Jin SW, Hanson C, Doty J, Jagaraj K, Braaten K, et al. Play it forward! A community-based participatory research approach to childhood obesity prevention. Fam Syst Health. 2016;34(1):15-30. https://doi.org/10.103 7/fsh0000116

11. Bodkin A, Hakimi S. Sustainable by design: a systematic review of factors for health promotion program sustainability. BMC Public Health. 2020;20(1):964. https://doi.org/10.1186/s12889-020-09091-9

12. Cairney J, Dudley D, Kwan M, Bulten R, Kriellaars D. Physical literacy, physical activity and health: toward an evidence-informed conceptual model. Sports Med. 2019;49(3):371-83. https://doi.org/10.1007/s40279-019-01063-3 .

13. Batterham R, Beauchamp A, Osborne R. Health literacy. In: The International Encyclopedia of Public Health: Academic Press; 2017. p. 428-37. https://doi. org/10.1016/B978-0-12-803678-5.00190-9

14. Tremblay MS, Longmuir PE, Barnes JD, Belanger K, Anderson KD, Bruner B, et al. Physical literacy levels of Canadian children aged 8-12 years: descriptive and normative results from the RBC learn to play-CAPL project. BMC Public Health. 2018;18(S2):1036. https://doi.org/10.1186/s12889-018-5891-x

15. Stadt Köln. Statistische Daten - Thematische Karte. https://www.stadt-koeln. de/politik-und-verwaltung/statistik/statistische-daten-thematische-karte. Accessed 26 Mar 2021.

16. Fernandez ME, Ruiter RAC, Markham CM, Kok G. Intervention mapping: theory- and evidence-based health promotion program planning: perspective and examples. Front Public Health. 2019;7:209. https://doi.org/1 0.3389/fpubh.2019.00209.

17. Kok G, Peters LWH, Ruiter RAC. Planning theory- and evidence-based behavior change interventions: a conceptual review of the intervention mapping protocol. Psicol Reflex Crit. 2017;30(1):19. https://doi.org/10.1186/ s41155-017-0072-x .

18. Schreier M. Qualitative content analysis in practice. Los Angeles, London, New Delhi, Singapore, Washington DC: SAGE; 2012.

19. Dahlgren $G$, Whitehead M. European strategies for tackling social inequities in health: Levelling up Part 2 2006: WHO Regional office for Europe Copenhagen. 
20. Liu Y, Chen S. Physical literacy in children and adolescents: definitions, assessments, and interventions. Eur Phys Educ Rev. 2021;27(1):96-112. https://doi.org/10.1177/1356336X20925502 .

21. Edwards LC, Bryant AS, Keegan RJ, Morgan K, Jones AM. Definitions, foundations and associations of physical literacy: a systematic review. Sports Med. 2017;47(1):113-26. https://doi.org/10.1007/s40279-0160560-7.

\section{Publisher's Note}

Springer Nature remains neutral with regard to jurisdictional claims in published maps and institutional affiliations.

Ready to submit your research? Choose BMC and benefit from:

- fast, convenient online submission

- thorough peer review by experienced researchers in your field

- rapid publication on acceptance

- support for research data, including large and complex data types

- gold Open Access which fosters wider collaboration and increased citations

- maximum visibility for your research: over $100 \mathrm{M}$ website views per year

At $\mathrm{BMC}$, research is always in progress.

Learn more biomedcentral.com/submissions 